\title{
Identification of a dynamic friction model and its application in a precise tracking control
}

\author{
Joanna Piasek, Radosław Patelski, Dariusz Pazderski, \\ Krzysztof Kozłowski
}

Institute of Automation and Robotics, Poznan University of Technology, ul. Piotrowo 3a, Poznań, Poland; joanna.z.piasek@ doctorate.put.poznan.pl, radoslaw.z.patelski@doctorate.put.poznan.pl,dariusz.pazderski@put.poznan.pl, krzysztof.kozlowski@put.poznan.pl

\begin{abstract}
The goal of this paper is to examine the friction behaviour in a one-degree mechanical system designed for precise tracking. Friction as one of the main disturbances present in this system strongly influences its performance, which is most visible during the velocity reversals. Identification and compensation of the friction are crucial to achieve high tracking accuracy at very low velocities. In this paper the procedure for identification of static and dynamic frictional parameters of LuGre is presented. The experimental results show characteristic behaviours of friction present both in sliding and in presliding regime. Furthermore, it is experimentally proven in several control scenarios that dynamic friction model compensation causes significant decrease of trajectory tracking error.
\end{abstract}

Keywords: friction dynamic models; friction identification; precise tracking; the active disturbance rejection paradigm; control of astronomic mounts

\section{Introduction}

One of the main disturbances deteriorating the quality of control of mechanical systems is friction. Its characteristics can be divided into two stages: presliding (or micro-slip) regime and sliding regime. Classical methods of friction analysis are focused on the description of the sliding regime. Such models represent the static relationship between speed and friction force. The simplest of them consists of Coulomb and viscous friction superposition. Other models take into account also the Stribeck effect, which brings a better approximation of the phenomena at low speeds, [2]. Still, in each of these cases, the presliding behaviour is not considered. It is well known, however, that motion controllers taking advantage of only classical friction models may provide unsatisfactory results, especially at zero-crossing instants of the velocity due to the discontinuity in the velocity-friction characteristics. Therefore, it is important to study more complex models, including also dynamic phenomena, such as friction hysteresis and micro-slips in the presliding regime. 
The problem of friction modelling becomes critical in applications where a precise control is required. Important examples of such applications can be found in astronomy. Nowadays, astronomical observations require often the use of mechanical and drive structures (so-called mounts, to which the telescope is attached) and control techniques known in robotics. One of the biggest challenges is to achieve precise trajectory tracking in the task space over a wide range of speed. Currently, at the Poznan University of Technology, an altitude-azimuth mounting structure for a $0.5 \mathrm{~m}$ diameter class telescope is being developed, [12]. It is expected that the device is able to track the objects at the sky at very low speeds in the order of several arcseconds per second (resulting from the daily rotation of the Earth on its axis) and larger ones reaching several degrees per second. Moreover, it is supposed to allow quick reconfiguration in order to shorten the time necessary to start the observation of the next object.

The paper covers two main topics. The first is focused on experimental identification of friction in a mechanical joint of the astronomic mount. The second deals with the application of friction models to design a tracking controller based on the active disturbance rejection (ADR) paradigm originally introduced by Han and Gao $[7,8]$. The considered control strategy enables an adaptation to unknown dynamic terms of the process using a high-gain observer, [11]. Thus, it is possible to consider the ADR as a particular implementation of the concept of free-model control, [5, 6]. However, in many applications observer gains cannot be increased arbitrarily. This is due to the presence of measurement noises, additional actuators dynamics as well as the delays in control loop, [14]. Then in order to improve control quality an ADR controller can be supplemented with more complex models of the process. Taking advantage of this possibility we design a motion controller and investigate if the application of friction models brings a relevant improvement in the tracking precision for the astronomic mount. We compare experimentally an impact of various models on the controller performance.

Recently, preliminary results of the friction identification of the considered mount have been already reported in [15]. In this paper, however, they are revised and significantly extended. To the best authors' knowledge the implementation of dynamic friction models in the ADR control scheme is original and has not been reported in the literature.

The paper is organized as follows. In Section 2 basic friction models are recalled. Section 3 is focused on the design of tracking controller using the ADR approach supported by friction models. In Section 4 an extensive experimental research is discussed. The results of the friction identification and the tracking control using different models are shown. The last Section concludes the paper.

\section{List of symbols}

All symbols are given in the order of appearance.
$J \quad$ moment of inertia
$q$ axis angular position
$h$ disturbances in the system

$$
\begin{aligned}
& {\left[\mathrm{kg} \cdot \mathrm{m}^{2}\right]} \\
& {[\mathrm{rad}]} \\
& {[\mathrm{Nm}]}
\end{aligned}
$$


friction torque

$[\mathrm{Nm}]$

$\tau$ control input, torque

$\sigma_{0} \quad$ stiffness coefficient of the friction model

[Nm]

$\sigma_{1} \quad$ damping coefficient of the friction model

$[\mathrm{Nm} / \mathrm{rad}]$

$[\mathrm{Nm} \cdot \mathrm{s} / \mathrm{rad}]$

$\sigma_{2} \quad$ viscous coefficient of the friction model

$V_{s} \quad$ Stribeck velocity

$F_{S} \quad$ Stribeck friction

$F_{c} \quad$ Coulomb friction

$\delta \quad$ empirical tuning coefficient of the friction model

$z \quad$ average displacement of bristles in the friction model

$\dot{q}, \omega$ axis angular velocity

$q_{d} \quad$ desired angular position

$\dot{q}_{d} \quad$ desired angular velocity

$\ddot{q}_{d} \quad$ desired angular acceleration

$e \quad$ tracking position error

$\dot{e} \quad$ tracking velocity error

$k_{p} \quad$ gain of proportional term of the tracking controller

$[\mathrm{Nm} \cdot \mathrm{s} / \mathrm{rad}]$

$[\mathrm{rad} / \mathrm{s}]$

$[\mathrm{Nm}]$

$[\mathrm{Nm}]$

$[\mathrm{rad}]$

$[\mathrm{rad} / \mathrm{s}]$

$[\mathrm{rad}]$

$[\mathrm{rad} / \mathrm{s}]$

$\left[\mathrm{rad} / \mathrm{s}^{2}\right]$

[rad]

$[\mathrm{rad} / \mathrm{s}]$

$k_{d} \quad$ gain of derivative term of the tracking controller

$w \quad$ estimate of total disturbance $\tau_{f}+h$

$[\mathrm{Nm} / \mathrm{rad}]$

$d \quad$ lumped disturbance, $d=-w+h+\tau_{f}$

$[\mathrm{Nm} \cdot \mathrm{s} / \mathrm{rad}]$

$[\mathrm{Nm}]$

$[\mathrm{Nm}]$

$[\mathrm{Nm}]$

$\tau^{*} \quad$ estimate of input reduced by friction, $\tau^{*}=\tau-\hat{\tau}_{f}$

$\hat{\tau}_{f} \quad$ estimate of friction force

$[\mathrm{Nm}]$

$[\mathrm{rad}]$

$[\mathrm{rad} / \mathrm{s}]$

$\zeta_{2}$ estimate of $\dot{q}$

$\zeta_{3}$ estimate of reduced lumped disturbance

$L \quad \mathbb{R}^{3}$ vector of obsever gains

\section{Friction models}

Let us consider the following dynamics of one-degree of freedom mechanical system

$J(q) \ddot{q}+h+\tau_{f}=\tau$,

where $q$ denotes a configuration, $J$ is a moment of inertia, $h$ describes a disturbance (including gravity, dynamic couplings between other links of a multi-body system, etc.), $\tau_{f}$ stands for friction and $\tau$ is a torque (force) input.

Further, we consider operation conditions in which inertial forces are negligible while friction $\tau_{f}$ constitutes a predominant term in dynamics (1). As a result, its proper modelling can be required for simulation analysis as well as control design. In the successive sections we briefly recall basic approaches to describe friction effects.

\subsection{Static model}

The static model is only dependent on the velocity $\omega=\dot{q}$. It describes the friction $\tau_{f}$ behaviour in the sliding state. The most common static model, taking into account 
the Stribeck effect, was presented by Armstrong in the following form, [2]:

$$
\tau_{f}=\sigma_{2} \omega+\operatorname{sign}(\omega)\left(F_{c}+\left(F_{s}-F_{c}\right) \exp \left(-\left|\frac{\omega}{V_{s}}\right|^{\delta}\right)\right) .
$$

The expression $\sigma_{2} \omega$ represents viscous friction force, while the rest of the equation describes Stribeck effect. This model is characterized by 5 parameters: static friction $F_{s}$, Coloumb torque/force $F_{c}$, Stribeck velocity $V_{s}$, shape coefficient $\delta$ and viscous friction coefficient $\sigma_{2}$.

The significant disadvantage of this model is the discontinuity by velocity reversals, what can cause errors and instability in the friction compensation procedure.

\subsection{Dahl model}

The Dahl model is one of the oldest friction models (1968), that describes behaviour of the friction in the presliding regime, that means in the stage when the input signal is not big enough to break the static friction force. In this regime, the rough structures building contact surfaces are getting deformed, resulting in micro-scale motion, [4]. These roughness form a system similar to a spring-system. When the external force is high enough, the spring breaks and the sliding movement begins. Dahl model takes into account the break-away moment of static friction $F_{s}$. It presents friction as a first order differential equation with respect to position $q$, [4]:

$\frac{d \tau_{f}}{d q}=\sigma_{0} \operatorname{sign}\left(1-\frac{\tau_{f}}{F_{s}}\right)\left|1-\frac{\tau_{f}}{F_{s}}\right|^{n}$.

The model consists of three parameters: micro-stiffness coefficient $\sigma_{0}$, static friction force $F_{s}$ and shape factor $n$.

\subsection{LuGre model}

LuGre model was presented by Canudas de Wit [3], as an extension of Dahl model by function describing the Stribeck effect. Both regimes are described using the same group of equations without use of a switching function, what results in a smooth transition between sliding and presliding regimes.

This model is based on a system of bristles (bristle model), where the internal state variable $z$ represents their average displacement. By usage of a first order differential equation authors described friction dynamic phenomena like frictional lag and presliding displacement. LuGre model consists of 7 parameters and is described below:

$$
\begin{aligned}
& \dot{z}=\omega-\sigma_{0} \frac{|\omega|}{s(\omega)} z \\
& s(\omega)=F_{c}+\left(F_{s}-F_{c}\right) \exp \left(-\left|\frac{\omega}{V_{s}}\right|^{\delta}\right), \\
& \tau_{f}=\sigma_{0} z+\sigma_{1} \dot{z}+\sigma_{2} \omega,
\end{aligned}
$$


For small deformations, the model behaves like a spring with stiffness $\sigma_{0}$ and damping coefficient $\sigma_{1}$. In the steady state, this model is reduced to a static model. Setting the micro-stiffness coefficient to $\sigma_{0}$ and micro-damping friction coefficient $\sigma_{1}$ to 0 and equaling Coulomb $F_{c}$ and Stribeck friction $F_{s}$ reduces the LuGre model to the Dahl model with shape factor $n=1$.

\section{Tracking controller with friction compensation}

\subsection{The general form of the control law}

Let $q_{d}$ be a reference trajectory which is smooth enough (at least the second order time derivative is bounded). Next, we define tracking error, $e:=q_{d}-q$, and consider a PD-like controller equipped with feed-forward and compensation terms, cf. [14],

$\tau=J(q)\left(\ddot{q}_{d}+k_{p} e+k_{d} \dot{e}\right)+w$,

where $k_{p}>0$ and $k_{d}>0$ are positive gains, and $w$ is an estimate of $\tau_{f}+h$.

Substituting (7) to (1) one can obtain the following closed loop system

$J(q) \ddot{e}=-J(q)\left(k_{p} e+k_{d} \dot{e}\right)+d$,

where $d:=-w+h+\tau_{f}$ denotes lumped disturbance which comes from a mismatch between the model and the real dynamics.

Now, assuming that the disturbance is at least locally bounded in a subset of the state-space where the system evolution takes place, one can expect that for any positive $k_{p}$ and $k_{d}$ tracking error converges to vicinity of zero, such that

$\lim _{t \rightarrow \infty}|e(t)| \leq C \sup _{t}|d(t)|$,

where $C$ is a positive constant, which is dependent on the chosen gains. Hence, one can conclude that for fixed values of parameters $k_{p}$ and $k_{d}$, the tracking accuracy can be improved by reducing the magnitude of disturbance $d$. Basically, such a reduction can be achieved in two ways: by using a more accurate model of the process or by application of adaptation techniques.

In this paper we combine two approaches. Specifically, in order to introduce an adaption mechanism we take advantage of a high gain extended state observer (ESO) and assume that a friction model becomes a part of the controller, while estimate of $h$ stays unknown. In such a case, the observer is designed based on the following dynamics

$\ddot{q}=\frac{1}{J(q)}\left(-h+\tau^{*}\right)$,

where $\tau^{*}:=\tau-\hat{\tau}_{f}$, while $\hat{\tau}_{f}$ stands for the assumed model of friction. Then the ESO observer can be designed as follows

$\dot{\zeta}=\left[\begin{array}{lll}0 & 1 & 0 \\ 0 & 0 & 1 \\ 0 & 0 & 0\end{array}\right] \zeta+\left[\begin{array}{c}0 \\ \frac{1}{J(q)} \tau^{*} \\ 0\end{array}\right]+L\left(q-\zeta_{1}\right)$, 
where $\zeta=\left[\zeta_{1} \zeta_{2} \zeta_{3}\right]^{\top}$ denotes estimates and $L \in \mathbb{R}^{3}$ defines the observer gains which are chosen based on the Routh-Hurwitz stability criterion. It can be proved that for $\sup _{t}|\dot{h}|<M_{1}$ and $\sup _{t}\left|\dot{\tau}_{f}-\dot{\hat{\tau}}_{f}\right|<M_{2}$, where $M_{1}, M_{2}$ are constants, estimate $\zeta$ satisfies the following

$\lim _{t \rightarrow \infty}\left|\zeta_{1}-q\right| \leq \varepsilon_{1}, \lim _{t \rightarrow \infty}\left|\zeta_{2}-\dot{q}\right| \leq \varepsilon_{2}, \lim _{t \rightarrow \infty}\left|\zeta_{3}-\frac{1}{J(q)}\left(\hat{\tau}_{f}-\tau_{f}-h\right)\right| \leq \varepsilon_{3}$,

with $\varepsilon_{1}, \varepsilon_{2}$ and $\varepsilon_{3}$ being positive constants which can be made small enough by increasing the observer gains, for example see $[18,1]$.

Consequently, one can consider $\zeta_{2}$ as an estimate of velocity $\dot{q}$, while $J(q) \zeta_{3}$ can be viewed as an approximation of the total disturbance. Following this observation, one can assume that $w$ in (7) satisfies

$w:=\hat{\tau}_{f}-J(q) \zeta_{3}$

and can be considered as an approximation of $h+\tau_{f}$. Then recalling definition of $d$ one can conclude that magnitude of this term can be attenuated. Alternatively, it can be stated that the disturbance is actively rejected in the control loop.

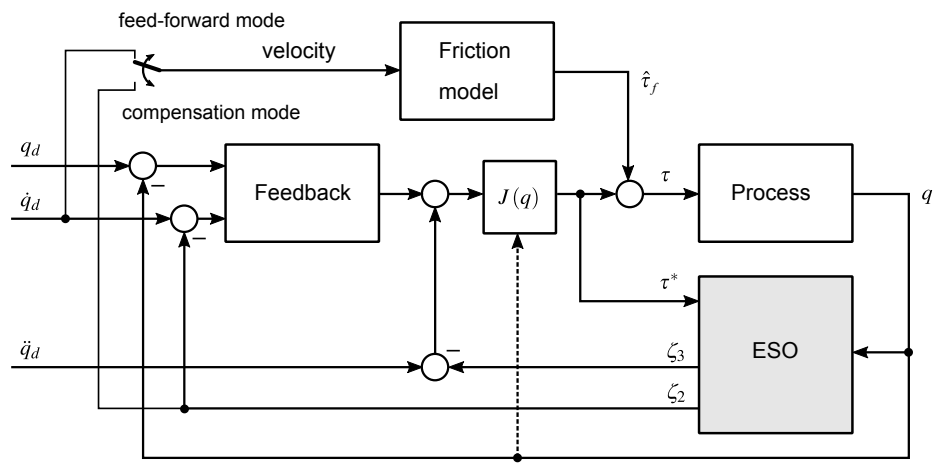

Figure 1

Diagram of the tracking controller with the friction model.

Remark. The stability of the closed loop system is considered under relatively strong assumptions. In particular, the requirements formulated with respect to time derivatives of $h, \tau_{f}$ and $\hat{\tau}_{f}$ could be seen as limiting factors. However, locally for the considered operation conditions, they can be justified. In particular, it is noteworthy that the application of dynamic friction models makes it possible to confirm that $\dot{\tau}_{f}$ and $\dot{\hat{\tau}}_{f}$ are bounded in a neighborhood of zero velocity.

\subsection{Friction compensation}

The outlined controller taking advantage of the ADR approach allows one to incorporate friction model $\hat{\tau}_{f}$ in different ways. Basically, a priori knowledge about the friction model may limit the process uncertainty that can lead to a better accuracy 
of estimation of residual disturbances. Particularly, it is expected that term $\left|\dot{\tau}_{f}-\dot{\hat{\tau}}_{f}\right|$ can be reduced which leads to the improvement of tracking performance.

The main problem considered in this paper can be briefly stated as follows.

Problem. We investigate the qualitative and quantitative aspects of using friction models in an ADR controller based on real experimental data.

In particular, we consider control tracking performance in the case of a slow-time varying trajectory determined for a robotic revolute joint and take into account the following approaches to define friction model $\hat{\tau}_{f}$ :

C1 Nominal case: the friction model is not considered, $\hat{\tau}_{f}:=0$,

C2 Compensation case: the friction model is computed based on the system state (i.e. velocity estimated by the observer), $\hat{\tau}_{f}:=\hat{\tau}_{f}(\hat{\dot{q}})$,

C2a Dynamic compensation case: full LuGre model is considered and dynamical effects are included based on the system state,

C2b Static compensation case: simplified static model is considered under assumption $\dot{z} \equiv 0$, based on the system state,

C3 Feed-forward case: the friction model is computed based on the reference trajectory (i.e. reference velocity $\left.\dot{q}_{d}\right), \hat{\tau}_{f}:=\hat{\tau}_{f}\left(\dot{q}_{d}\right)$,

C3a Dynamic compensation case: full LuGre model is considered and dynamical effects are included based on the reference trajectory,

C3b Static compensation case: simplified static model is considered under assumption $\dot{z} \equiv 0$, based on the reference trajectory.

The controller diagram with the friction model employed in the compensation or feed-forward path is presented in Fig. 1.

\section{Experimental work}

The experiments have been conducted using telescope mount developed at Institute of Automatic Control and Robotic of Poznan University of Technology, [12]. The studied object consists of a two-axis altitude-azimuth gearless robotic platform with an astronomic telescope with a mirror of diameter $0.5 \mathrm{~m}$. It is driven by permanentmagnet synchronous motors (PMSMs) which are capable of delivering torque of the order of $50 \mathrm{Nm}$. The measurement of angular positions is performed by two sets of four absolute encoders with a resolution of 32 bits, [9], while speed is estimated by numerical differentiation of the position signal. Control algorithms have been implemented in $\mathrm{C}++$ using Texas Instruments AM4379 Sitara processor with ARM Cortex-A9 core. The controller itself is implemented in a cascade form which consists of independent current and position loops. Both loops work simultaneously with frequency of $10 \mathrm{kHz}$.

The results reported in this paper have been obtained for the vertical axis of the mount which supported by a ball bearing. Since the identification procedure is 
performed offline, in this report we have presented only the most representative results among a wide number of experimental trials. All the measurements used in the identification scheme have been collected under normal operating conditions (after the system has heated up, in constant temperature, and the fixed position of the horizontal axis). Value of the moment of inertia $J=30 \mathrm{~kg} \cdot \mathrm{m}^{2}$ was estimated based on identification experiments and CAD model of the device.

The identification experiments of static friction as well as experiments investigating the controller performance have been conducted in the closed loop control regime with $k_{p}=255$ and $k_{d}=30$ what corresponds to bandwidth $\omega_{c}=15$ and damping coefficient $\zeta=1$ of PD regulator. The bandwidth of ESO observer was chosen as $\omega_{o}=220$, cf. also [14]. Identification of dynamic parameters has been carried out in an open loop. Both identification experiments are outlined in Figs. 2 and 3.

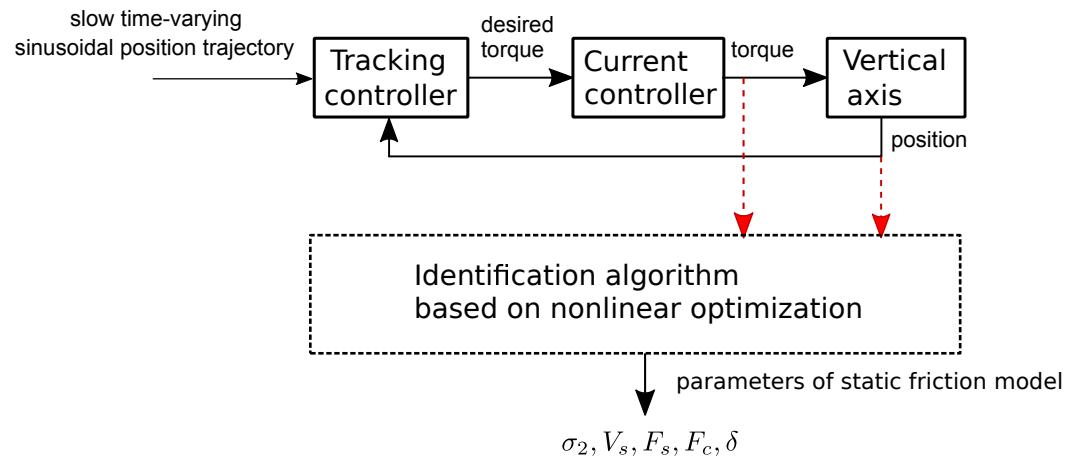

Figure 2

Diagram of the identification process of the static friction model in the closed loop regime (the case of a quasi-static excitation)

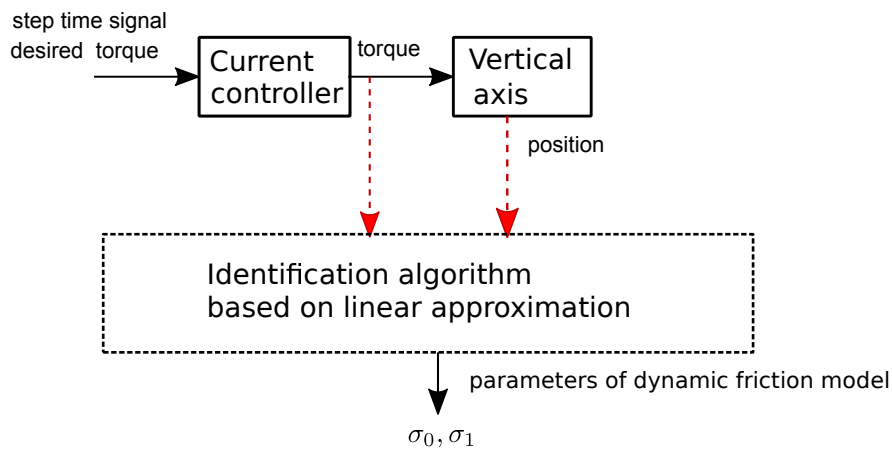

Figure 3

Diagram of the identification process of the dynamic friction model in the open loop regime (the case of a dynamic excitation) 


\subsection{Identification of friction model}

\subsubsection{Friction - velocity map}

The most common way to find the friction-velocity map is by registration of the input torque at constant velocity in the full range of available velocities $[13,16,3]$. Due to limitations connected with the long-lasting recording process, it was very difficult to conduct the corresponding experiment. Furthermore, PMSM drives generate torque with unwanted ripples, resulting from the construction of the motor. This entails the need for a comprehensive analysis of movement - preferably averaging measurements of a few revolutions of a telescope. Taking into account the geometric constraints imposed on the considered system it was impossible to repeat the experiments reported for other mounts, [13].

Alternatively, in order to find a friction-velocity map one can measure the velocity of the mount as a response for triangular or sine input of very low frequency and amplitude bigger than break-away torque required to just initiate the motion. This solution was presented in [17] and [10].

Quasi-static excitation was achieved using reference trajectory $q_{d}$ a such that $\dot{q}_{d}$ defines a sine wave of amplitude $0.056 \mathrm{rad} / \mathrm{s}$ and frequency $0.04 \mathrm{~Hz}$. The chosen stimulation minimizes the influence of mechanical dynamics, therefore the characteristics obtained in this way can be presented as quasi-static. To be more precise, recalling (1) in the considered conditions it is assumed that $|J(q) \ddot{q}|+|h|<<\left|\tau_{f}\right|$. Hence, the following approximation can be justified: $\tau_{f} \approx \tau$.

Nonlinear optimization was performed in Matlab (Curve Fitting Tool) to find static parameters based on the torque-velocity data by minimizing cost function:

$\min _{\sigma_{2}, V_{s}, F_{s}, F_{c}, \delta} \sum_{i=1}^{n}\left[\tau_{f i}-\hat{\tau}_{f i}\right]^{2}$

where $\hat{\tau}_{f}$ stands for the torque computed by the model.

Table 2

Estimated static parameters of LuGre model in the vertical axis

\begin{tabular}{|l|l|l|l|l|}
\hline Parameter & $\omega>0$ & $\omega<0$ & nominal case & unit \\
\hline$\sigma_{2}$ & 28.64 & 22.5 & 32.77 & $\mathrm{Nm} \mathrm{s} / \mathrm{rad}$ \\
\hline$F_{c}$ & 3.128 & 2.123 & 2.322 & $\mathrm{Nm}$ \\
\hline$F_{s}$ & 4.32 & 3.081 & 3.322 & $\mathrm{Nm}$ \\
\hline$V_{s}$ & 0.01129 & 0.02126 & 0.04129 & $\mathrm{rad} / \mathrm{s}$ \\
\hline$\delta$ & 1.2914 & 1.9 & 2 & - \\
\hline
\end{tabular}

Estimated values are collected in Table 2. From the obtained results one can conclude that friction characteristics are not symmetrical for two directions of motion. Figures $4 \mathrm{a}$ and $4 \mathrm{~b}$ show static friction parameters identification results for positive and negative velocities as well as for the nominal case, Fig. 4c, that collects results of all data independently of the velocity sign. The break-away torque takes smaller values when system velocity decreases than when it increases. The width of hysteresis loop grows with the acceleration increase. Stribeck effect in this system is 
negligible, which can be partially explained by the application of rolling bearings (instead of plain bearings).

a)

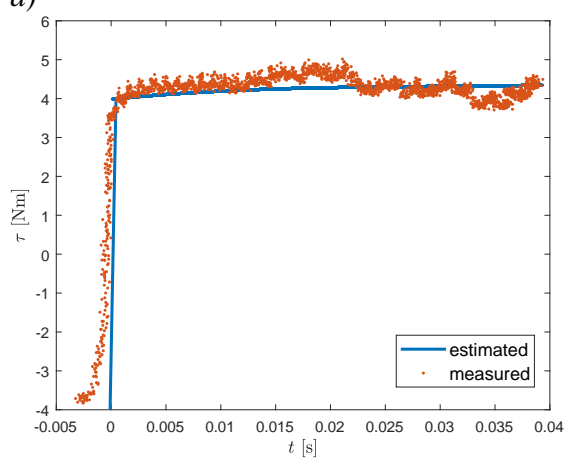

c)

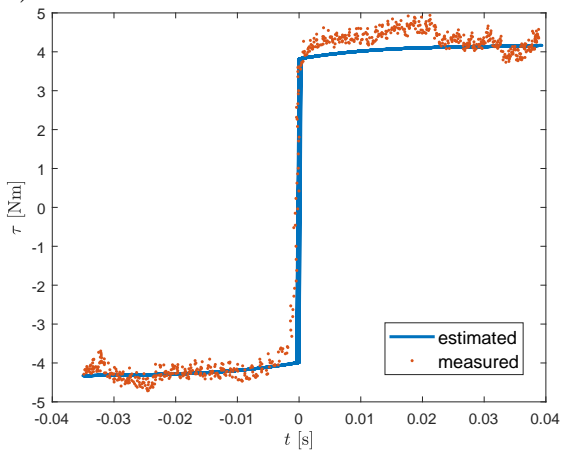

b)

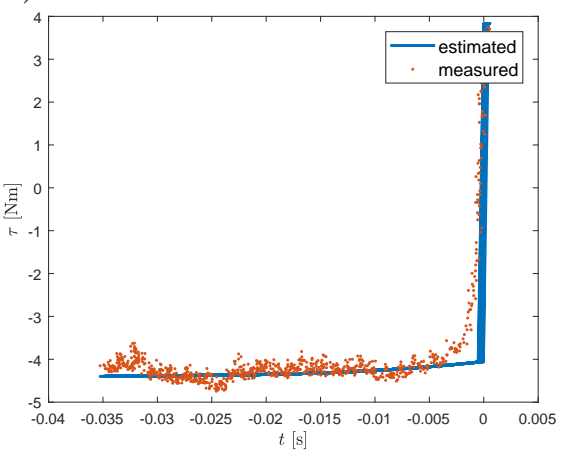

d)

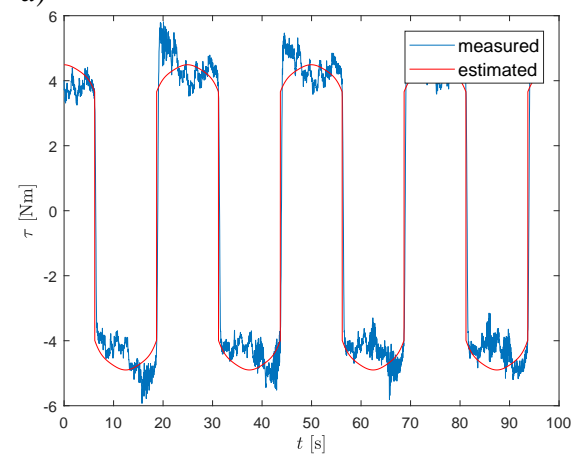

Figure 4

Static friction estimation when (a) $\omega>0$ (b) $\omega<0$ (c) nominal case (d) comparison of nominal static friction model with an input signal

From Fig. 5a one can conclude that the system behaviour in the open loop is not consistent, especially in the context of break-away friction torque, despite periodic input torque signal. Such properties of the object were not observed in any of the cited papers. This probably means that the assumed friction model does not reflect additional disturbances in the analyzed system. In Fig. 5b friction-velocity map for following periods are presented, the difference between curves reaches $2 \mathrm{Nm}$ for the corresponding velocities, this property causes variability of static friction parameters and shows that system is non-stationary.

\subsubsection{Dynamical parameters}

Estimation of dynamic parameters values $\sigma_{0}$ and $\sigma_{1}$ is not possible by direct usage of linear estimation techniques because of the non-linear relationship between friction and these parameters and the impossibility of measuring the variable $z$, that represents bristle displacement due to junctional deformations at the surface interface. Instead, a simplified method based on the linear approximation of the system 
a)

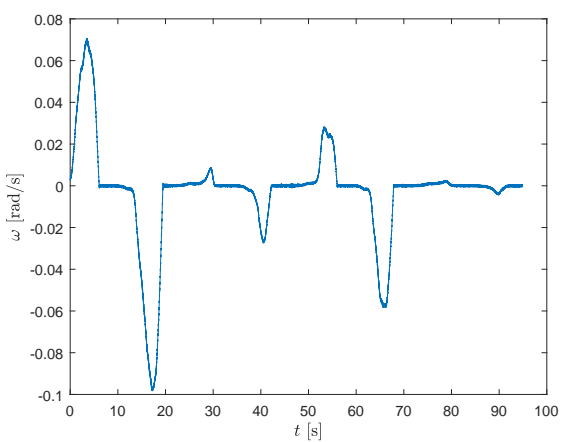

b)

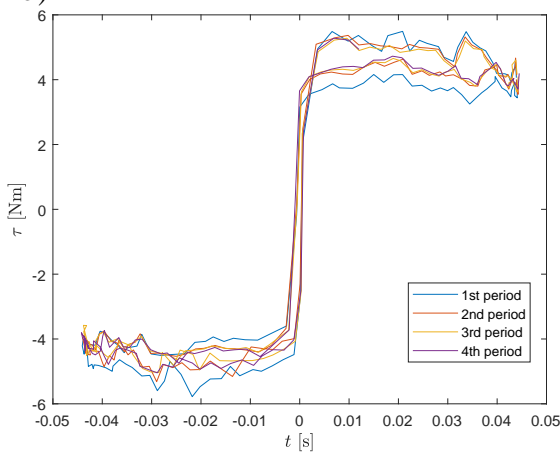

Figure 5

(a) system's response for the periodic input torque signal that results in varying break-away friction. (b) velocity-friction map in the closed loop system for several sine periods

in the presliding stage can be applied. Basically, when torque $\tau$ is less than the breaking torque, Eq. (4) reduces to $\dot{z}=\omega$ and the essential system dynamics can be described by:

$J \ddot{q}+\left(\sigma_{1}+\sigma_{2}\right) \dot{q}+\sigma_{0} q=\tau$.

The corresponding transfer function is as follows:

$$
\frac{Q(p)}{U(p)}=\frac{\frac{1}{\sigma_{0}}}{\frac{J}{\sigma_{0}} p^{2}+\frac{\sigma_{1}+\sigma_{2}}{\sigma_{0}} p+1},
$$

where $p$ is a complex variable, $Q(p)$ and $U(p)$ stand for Laplace transforms of $q$ and $\tau$, respectively.

In the applied identification procedure, estimation of dynamic parameters $\sigma_{0}$ and $\sigma_{1}$ abridges to parameters estimation of the transfer function $\frac{Q(p)}{U(p)}$, for example by analyzing the system response for an input step of small amplitude (smaller than the break-away torque).

In the conducted experiments the desired torque defined by a step function of value of $\pm 1 \mathrm{Nm}$ (the function jumps from 0 to \pm 1 , respectively) was employed. Estimated parameters are presented in Table 3, measured and estimated step responses for velocities of different signs are presented in Fig. 6. The resulted characteristics are not symmetrical.

Table 3

Estimated dynamic parameters in the vertical axis

\begin{tabular}{|l|l|l|l|}
\hline & $\omega>0$ & $\omega<0$ & unit \\
\hline$\sigma_{0}$ & $9.7869 \cdot 10^{4}$ & $8.674 \cdot 10^{4}$ & $\mathrm{Nm} / \mathrm{rad}$ \\
\hline$\sigma_{1}$ & 521.4151 & 473 & $\mathrm{Nm} \mathrm{s} / \mathrm{rad}$ \\
\hline
\end{tabular}


a)

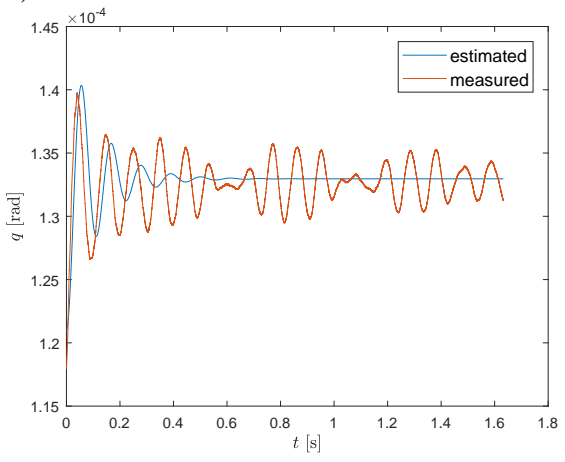

b)

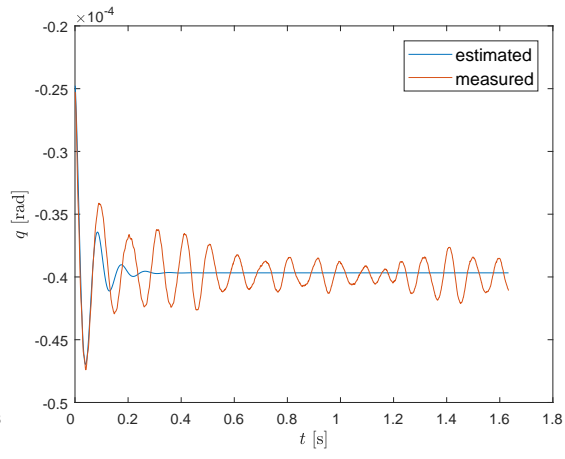

Figure 6

Dynamic friction estimation: (a) $\omega>0$, (b) $\omega<0$

\subsection{Tracking control}

\subsubsection{Implementation of friction model}

Typically, friction effects are described by fast dynamics. As a result, value of parameter $\sigma_{0}$ in (4) is large, which makes numerical integration of the friction model challenging. In such a case (4) can be considered as a stiff differential equation. In order to overcome this issue, we assume that velocity $\omega$ is a slow-time varying function. In particular, for $\dot{\omega}=0$ one can find the following approximated analytic solution of (4)

$z(t)=\left(1-\exp \left(-\frac{|\omega| \sigma_{0}}{s(\omega)} t\right)\right) \frac{\operatorname{sign}(\omega) s(\omega)}{\sigma_{0}}+\exp \left(-\frac{|\omega| \sigma_{0}}{s(\omega)} t\right) z(0)$,

where $z(0)$ stands for the initial condition. This result has been used to implement the friction model in the discrete time domain, namely a new value of $z$ has been computed at each time interval for the given value of $\omega$ from $t$ to $t+T_{s}$, where $T_{s}=0.1 \mathrm{~ms}$ is the sampling time.

\subsubsection{Friction compensation}

Having obtained both the identified parameters of the friction model and the numerical implementation of differential equations in the discrete time domain, series of experiments have been undertaken to investigate practical usability of LuGre model in a task of high-precision tracking control. Each of the cases defined in section 3.2 has been separately implemented and tested. During every experiment the desired trajectory was chosen as a sine wave with frequency of $f_{d}=\frac{1}{5} \mathrm{~Hz}$ and maximum velocity of $\omega_{d \max }=5 \omega_{s}$, where $\omega_{s}=7.268 \cdot 10^{-5} \frac{\mathrm{rad}}{\mathrm{s}}$ stands for the velocity of stars observed on the night sky.

Tracking error $e(t)$, friction force $\hat{\tau}_{f}(t)$ computed from the implemented model, torque $\tau_{d}$ produced by the controller (for the real control system torque $\tau$ is achieved indirectly taking advantage of an auxiliary current controller - here for simplicity it is assumed that $\tau=\tau_{d}$, cf. [14]) and an estimate of the total disturbance represented 
Table 4

Values of Integral Square Error criterion calculated for each experiment

\begin{tabular}{|c|c|c|c|c|c|}
\cline { 2 - 5 } \multicolumn{1}{c|}{} & $\mathrm{C} 1$ & $\mathrm{C} 2 \mathrm{a}$ & $\mathrm{C} 2 \mathrm{~b}$ & $\mathrm{C} 3 \mathrm{a}$ & $\mathrm{C} 3 \mathrm{~b}$ \\
\hline ISE criterion $[\operatorname{arcsec}]$ & 47.58 & 12.75 & 73.71 & 9.29 & 41.29 \\
\hline
\end{tabular}

by $\zeta_{3}(t) J(q)$ and provided by the extended state observer have been recorded during each experiment. All measures were registered after a short initial stage when an auxiliary trajectory was generated in order to ensure a smooth transition from the motionless state. Thus, for $t \geq 0$ a discontinuity in the desired trajectory $t=0$ is avoided. Obtained results are presented in Figs. 7-11. Integral square error criterion within time horizon $T_{h}=15 \mathrm{~s}$ has been calculated for each of the experiments and the corresponding values are presented in Tab. 4.

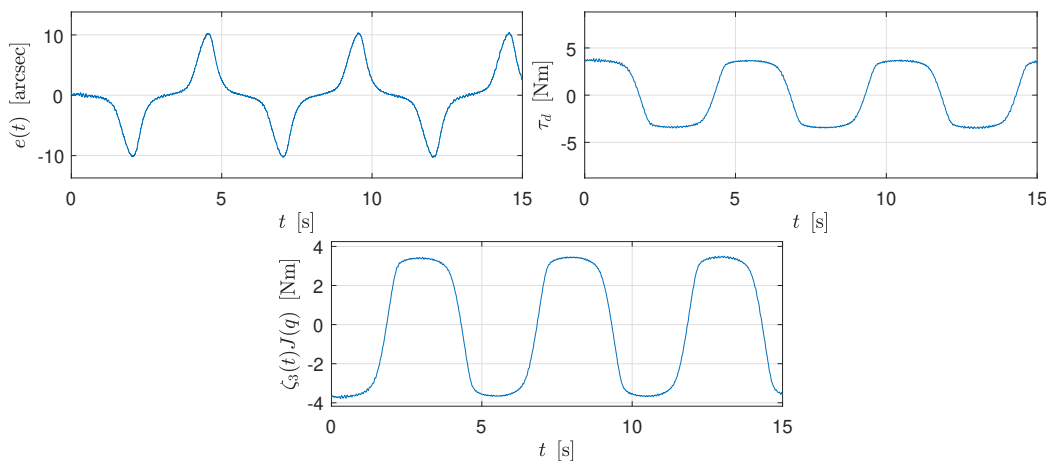

Figure 7

Tracking error, desired torque and estimated disturbance during $\mathrm{C} 1$ experiment
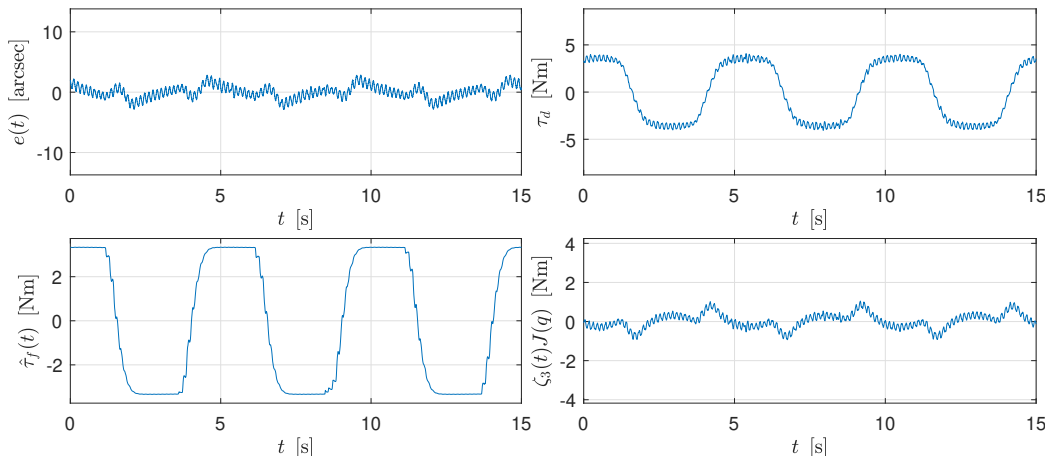

Figure 8

Tracking error, calculated friction force, desired torque and estimated disturbance during C2a experiment

Several conclusions can be drawn from the presented figures. The effectiveness of friction compensation using dynamic LuGre model is successfully confirmed by a significant decrease of the tracking error, especially in intervals where the sign of velocity changes. Over $80 \%$ decrease of ISE criterion was obtained by using the 

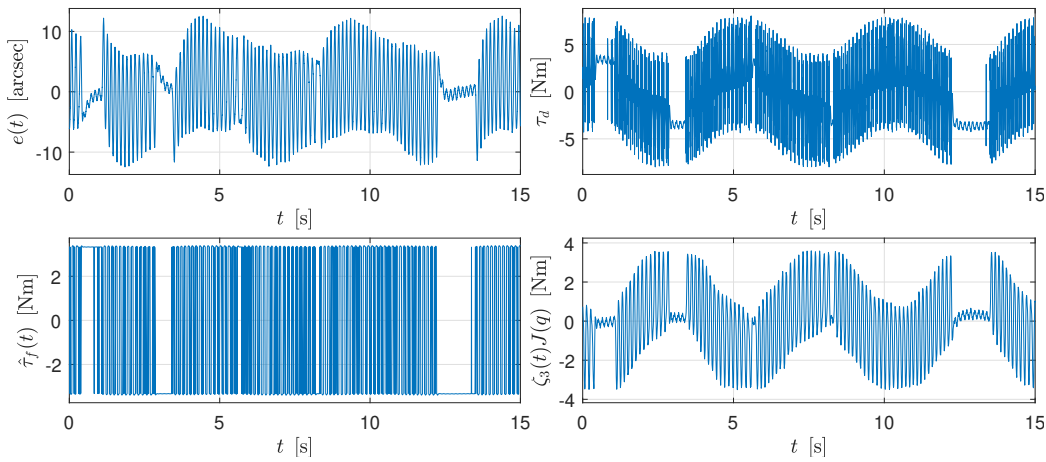

Figure 9

Tracking error, calculated friction force, desired torque and estimated disturbance during $\mathrm{C} 2 \mathrm{~b}$ experiment
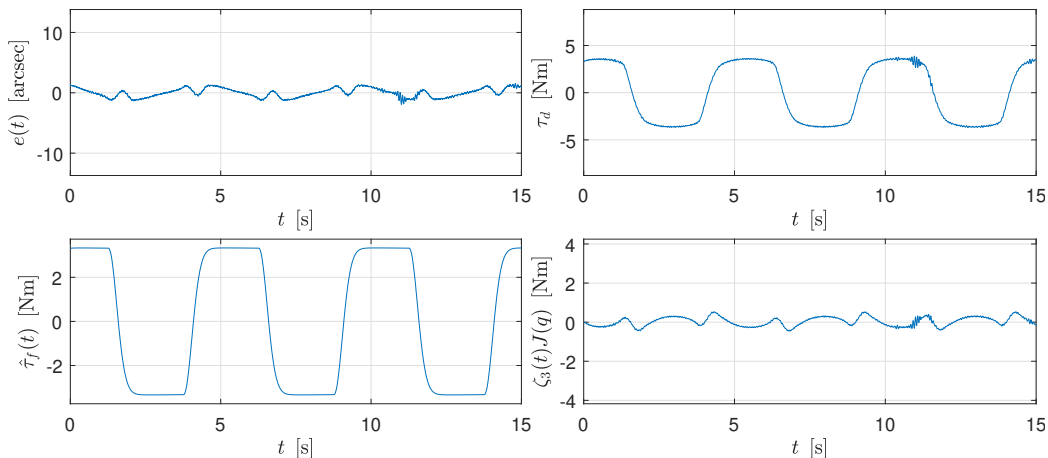

Figure 10

Tracking error, calculated friction force and estimated disturbance during C3a experiment

feed-forward compensation based on the desired velocity and almost 75\% decrease by usage of the feedback variant based on the estimated velocity of the joint. Commonly used feed-forward compensation using static only friction model proved to be unable to significantly improve the tracking quality while feedback-based static model led to increase of tracking errors due to the noise present in the velocity estimate. Thus, it can be expected that the low-pass filtering properties of LuGre model dynamics are able to restore the usability of a disturbed velocity signal. Moreover, from a comparison of the disturbance torque in Fig. 7 and inverted (due to the definition of disturbance in (8)) friction torque obtained from the models in the other experiments, one can conclude about quality of chosen friction model, identified parameters and presence of other unmodelled dynamic effects in the system. The similarity of the compared signals suggests that friction is indeed the main disturbing torque in the considered telescopic mount and that model parameters have been, at least locally, identified correctly.

\section{Conclusions}

The aim of this study is to investigate the phenomenon of friction in a robotic system with application to precise motion control. Based on the experimental data dynamic 

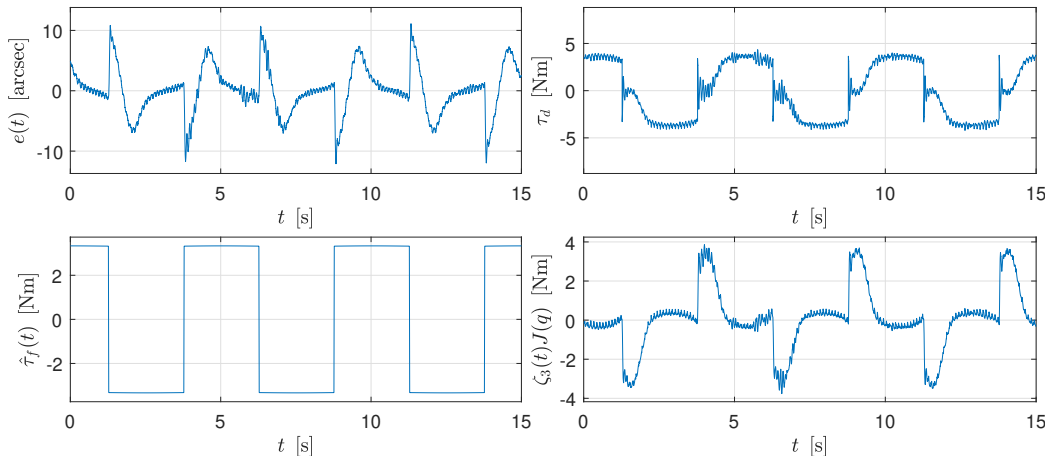

Figure 11

Tracking error, calculated friction force, desired torque and estimated disturbance during $\mathrm{C} 3 \mathrm{~b}$ experiment

friction model has been identified properly, however, the lack of symmetry with respect to the sign of the velocity has been noticed. Moreover, the studied object is strongly nonstationary.

In spite of these limitations, it was observed that a dynamic friction model makes it possible to improve the tracking precision when reversal motion in a revolute joint with by a ball bearing is considered. Based on the experimental data, the best results for the proposed ADR-based controller are achieved when LuGre friction model is used in the feed-forward path. In contrast, static friction models seem to be inappropriate for the considered motion conditions. It is noteworthy to emphasize that model inaccuracies are effectively attenuated by the ESO observer at least for slow time-varying disturbances. However, in order to significantly improve the tracking performance, it is necessary to accurately model fast disturbances which cannot be precisely estimated by the observer due to limited bandwidth.

In future works, it may be interesting to enrich deterministic friction models with stochastic models. Additionally, the high variability of friction depending on parameters such as temperature shows on-line identification can be required to improve robustness of the controller. Alternatively, more complex dynamics of the friction can be implemented.

\section{Acknowledgement}

This work was supported by the National Science Centre (NCN) under the grant No. 2014/15/B/ST7/00429, contract No. UMO-2014/15/B/ST7/00429.

\section{References}

[1] C. Aguilar-Ibanez, H. Sira-Ramirez, and J.A. Acosta. Stability of active disturbance rejection control for uncertain systems: A Lyapunov perspective. International Journal of Robust and Nonlinear Control, 27(18):4541-4553, 2017.

[2] B. Armstrong-Hélouvry. Control of machines with friction. Norwell, MA: Kluwer Academic Publishers, 1991. 
[3] C. Canudas-de Wit, H. Olsson, K.J. Astrom, and P. Lischinsky. A new model for control of systems with friction. IEEE Transactions on Automatic Control, 40(3):419-425, 1995.

[4] P. R Dahl. Measurement of solid friction parameters of ball bearings. Technical report, Aerospace Corp El Segundo Ca Engineering Science Operations, 1977.

[5] M. Fliess and C. Join. Model-free control and intelligent PID controllers: Towards a possible trivialization of nonlinear control? IFAC Proceedings Volumes, 42(10):1531-1550, 2009. 15th IFAC Symposium on System Identification.

[6] M. Fliess and C. Join. Model-free control. International Journal of Control, 86(12):2228-2252, December 2013.

[7] Z. Gao. From linear to nonlinear control means: A practical progression. ISA Transactions, 41(2):177 - 189, 2002.

[8] J. Han. From PID to Active Disturbance Rejection Control. IEEE Transactions on Industrial Electronics, 56(3):900-906, March 2009.

[9] D. Janiszewski and M. Kiełczewski. Kalman filter sensor fusion for multi-head position encoder. In 2017 19th European Conference on Power Electronics and Applications (EPE'17 ECCE Europe), pages P.1-P.7, Sep. 2017.

[10] M.R. Kermani, Rajnikant V. Patel, and M. Moallem. Friction identification and compensation in robotic manipulators. IEEE Transactions on Instrumentation and Measurement, 56(6):2346-2353, 2007.

[11] H.K. Khalil and L. Praly. High-gain observers in nonlinear feedback control. International Journal of Robust and Nonlinear Control, 24(6):993-1015, 2014.

[12] K. Kozłowski, D. Pazderski, B. Krysiak, T. Jedwabny, J. Piasek, S. Kozłowski, S. Brock, D. Janiszewski, and K. Nowopolski. High precision automated astronomical mount. In R. Szewczyk, C. Zieliński, and M. Kaliczyńska, editors, Automation 2019. Advances in Intelligent Systems and Computing, volume 920. Springer, 2020.

[13] T.S. Kumar and R.N. Banavar. Identification of friction in the $50 / 80 \mathrm{~cm}$ aries schmidt telescope using the LuGre model. IFAC Proceedings Volumes, 44(1):980-985, 2011.

[14] R. Patelski and D. Pazderski. Tracking control for a cascade perturbed control system using the active disturbance rejection paradigm. Archives of Control Sciences, 29(2):387-408, 2019.

[15] J. Piasek, K. Kozłowski, and D. Pazderski. Identification of friction in a robotic astronomical 0.5-m telescope mount. In Proc. of IEEE International Conference on Intelligent Engineering Systems (INES) 2019 (To appear), 2019. 
[16] C.H. Rivetta and S. Hansen. Friction model of the 2.5-mts sdss telescope. In Telescope Control Systems III, volume 3351, pages 466-478. International Society for Optics and Photonics, 1998.

[17] M. Ruderman, F. Hoffmann, J. Krettek, J. Braun, and T. Bertram. Robust identification of nonlinear frictional dynamics for advanced controller design. IFAC Proceedings Volumes, 42(10):474-479, 2009.

[18] S. Shao and Z. Gao. On the conditions of exponential stability in active disturbance rejection control based on singular perturbation analysis. International Journal of Control, 90(10):2085-2097, 2017. 